\title{
LAS CARACTERÍSTICAS DEL DESAYUNO EN ALUMNOS DE EDUCACIÓN OBLIGATORIA
}

\section{BREAKFAST CHARACTERISTICS IN THE MANDATORY EDUCATION}

\author{
Muñoz Carrillo, J.C. ${ }^{1}$, Ruiz Dí az, A. ${ }^{1}$, Hernández Ruiz, A. ${ }^{1}$, Córdoba Ruiz, I. ${ }^{1}$
}

${ }^{1}$ Universidad de Murcia, España.

Recibido: Julio/2013 - Aceptado: Noviembre/2013.

\begin{abstract}
Resumen
El objetivo del presente estudio fue conocer las características del desayuno de los alumnos de educación obligatoria (tiempo, tipo de alimentos, compañía). La muestra estuvo compuesta por 345 sujetos, 223 alumnos de tercer ciclo de primaria y 122 alumnos de segundo ciclo de la E.S.O. El instrumento utilizado para la recogida de datos fue un cuestionario sobre las características del desayuno. Los resultados muestran que los niños/as de tercer ciclo de primaria desayunan mejor que los niños/as de segundo ciclo de la E.S.O. Siguiendo esta línea los niños desayunan mejor que las niñas en la etapa de educación obligatoria. El tiempo medio dedicado al desayuno es de 9 minutos y 31 segundos el sitio preferido para hacerlo es en la cocina acompañados por los padres y hermanos.
\end{abstract}

Palabras clave: Nutrición; primaria; secundaria; tiempo; compañía.

\section{Abstract}

The aim of this study was to determine the breakfast of compulsory education students (time, type of food, company). The sample consisted of 345 subjects, 223 students of third cycle primary and 122 students of second cycle of ESO The instrument used for data collection was a questionnaire on the characteristics breakfast. The results show that children / as primary graduate breakfast better than children / as second cycle of ESO Following this line of breakfast children better than girls at the stage of compulsory education. The average time for breakfast is 9 minutes and 31 seconds to make it a favorite place is in the kitchen accompanied by parents and siblings.

Keywords: Nutrition; primary; secondary; time; company.

\section{INTRODUCCIÓN}

El desayuno es considerado la comida más importante del día ya que es la primera que se realiza después de estar durmiendo durante una media de 8 horas. La ración ideal de desayuno ha suscitado un gran interés científico en el ámbito de la nutrición, especialmente el papel que desempeña en el equilibrio nutricional de los niños.

Los expertos señalan que para que tenga las mejores cualidades nutricionales debe incluir: un lácteo (un vaso de leche, un yogur, queso); cereales (preferiblemente pan, pero también copos de cereales, galletas, magdalenas, bizcochos); una fruta o zumo y una grasa de complemento (mejor aceite de oliva, pero también mantequilla, margarina). Por tanto no es recomendable la bollería industrial, zumos con mucho azúcar, bebidas con gas o estimulantes o abusar de las mantequillas o las mermeladas con azúcar. De igual modo señalan que variación resulta clave, porque no todos los alimentos del mismo grupo contienen las mismas propiedades por eso es importante lograr un equilibrio en el aporte de macro/micronutrientes (Galiano y Moreno, 2010).

Este desayuno representa entre $25 \%$ y el $30 \%$ del aporte energético necesario a lo largo del día, por lo que tiene más importancia en etapas de crecimiento. En concreto en España los niños y adolescentes se enfrentan a jornadas lectivas de 6 horas de media y a actividades físicas con una considerable demanda metabólica.

En este sentido Amat, Anuncibay, Soto, Alonso, Villalmanzo, Lopera (2006) consideran que la preadolescencia es una etapa fundamental para el desarrollo de los niños. En las niñas empieza entre los $8 \mathrm{y}$ los 13 años y en los niños entre los 12 y los 17 años.

Revisando estudios ya realizados en Espańa, se puede apreciar que el 88\% de los nińos y adolescentes sí desayunan aunque no siguen el modelo ideal del mismo. Las razones más dadas que llevan a los niños a no realizar un buen desayuno es que no ingieren los tres componentes básicos de un desayuno: lácteos, cereales y fruta, faltándoles uno o hasta incluso dos de ellos (Galiano y Moreno, 2010).

También hay que tener en cuenta que en numerosas ocasiones no se hace correctamente el desayuno por la falta de tiempo. Lo adecuado sería que los niños y jóvenes cenaran pronto y se fueran a la cama sin preocupaciones y con todo preparado (material escolar, ropa o uniforme, etc.), y evitar así la falta de tiempo para realizar el desayuno. Por lo tanto no le quitarían tiempo al desayuno en detrimento de otros menesteres, como puede ser preparar el material escolar. Aparte de ingerir lácteos, cereales y fruta también hay que tener muy en cuenta que el desayuno se debería de hacer estando presente todos los integrantes de la familia, especialmente los padres, los cuáles deberían de dar ejemplo. El desayuno debería de durar entre 15 y 20 minutos y realizarse en un ambiente adecuado y rodeado de los familiares (Amat et al., 2006).

La incorrecta realización del desayuno puede conllevar consecuencias negativas en el día a día de los jóvenes y en un futuro. Donde más se va a notar esta deficiencia es en el rendimiento escolar, ya que diversas investigaciones han demostrado que las calificaciones 
medias cambian en función de la calidad del desayuno. $\mathrm{Al}$ realizar un buen desayuno, el rendimiento escolar aumenta mientras que al realizar un mal desayuno las notas se ven perjudicadas (Herrero y Fillat 2006).

Sin embargo a largo plazo realizar un mal desayuno tiene consecuencias más negativas, como es la posibilidad de padecer obesidad. Se ha podido ver la relación entre el incremento del índice de masa corporal de los niños debido a una omisión del desayuno. El desayunar diariamente y más si se trata de una ingesta rica en Hidratos de carbono produce efectos positivos ya que es la principal fuente de energía del cuerpo humano. Facilita la secreción de hormonas que provocaran una sensación de satisfacción y ayuda a la producción de ácidos grasos de cadena corta que provocan un efecto saciante (Galiano y Moreno 2010).

Por lo tanto se puede señalar que realizar un adecuado desayuno teniendo en cuenta estas premisas puede repercutir de una manera positiva, mejorando la calidad global de la dieta y evitando posibles problemas de salud a largo plazo.

Los objetivos planteados para la investigación han sido: a) conocer si desayunan mejor los jóvenes de tercer ciclo de primaria o los de educación obligatoria secundaria; b) averiguar el tiempo dedicado a la ingesta de los alimentos y la frecuencia semanal con la que se realiza; c) conocer el lugar donde ingieren los alimentos; d) saber con quién o quienes realizan el desayuno; e) discernir cuáles son los motivos por lo que no realizan bien el desayuno; f) observar si desayunan mejor los chicos o las chicas y en que ciclo de educación obligatoria desayunan mejor.

\section{MÉTODO}

El presente estudio se llevó a cabo en 4 centros escolares de la Región de Murcia con alumnos que se encontraban estudiando el tercer ciclo de primaria y el segundo ciclo de la Educación Obligatoria Secundaria, bajo la Ley Orgánica de Educación (L.O.E) 2/2006 del 3 de mayo publicada en el Boletín Oficial del Estado (B.O.E) de 20 de abril de 2006.

Se ha definido como muestra de objeto de estudio 345 alumnos de los cuales 223 eran de tercer ciclo de primaria y 122 de segundo ciclo de la E.S.O de 4 centros educativos de la Región de Murcia. Han participado aquellos alumnos, que trajeron una autorización de consentimiento por parte de los padres madres o tutores. En total fueron 339 alumnos de los cuáles 25 fueron considerados nulos por no cumplir con todos los requisitos mínimos a la hora de rellenar los datos del cuestionario (completar más del $80 \%$ de las preguntas). $O$ por responder a todo el cuestionario después de contestar de forma negativa la pregunta de filtro.

La muestra estuvo compuesta por 196 sujetos masculinos y 149 femeninos. La principal característica de los alumnos participantes fue que tenían una media de edad de 12,5 años.

Los instrumentos utilizados fueron el "Cuestionario para estudiantes de tercer ciclo de primaria" (ANEXO 1) y el "Cuestionario para estudiantes de segundo ciclo de secundaria" (ANEXO 2), los cuales pasaron por cuatro fases: fase de diseño, fase de validación y fiabilidad, fase de recogida de datos y fase de transcripción de los datos obtenidos a una hoja de datos.

En la fase de diseño el instrumento de observación tuvo que ser elaborado empezando desde su comienzo, ya que no se pudo encontrar ningún cuestionario que reuniera las características necesarias para el estudio.

En la segunda fase se realizó un proceso de validación para el cual se ha considerado como jueces expertos a 6 endocrinos de distintos hospitales de la Región de Murcia con más de 10 años de experiencia.

En la tercera fase se pasó el cuestionario a los alumnos de quinto, sexto de primaria y tercero, cuarto de secundaria. El cuestionario fue totalmente anónimo y fue entregado a todos los alumnos que trajeron las autorizaciones con el consentimiento de los padres, madres o tutores y previo permiso de los directores de los centros escolares.

En la última fase se creó una hoja de Excel (hoja de registro) en la cual se introdujeron los datos obtenidos por partes de los alumnos tras rellenar el cuestionario. Para finalizar se realizó una estadística descriptiva, registrando medias y porcentajes a través de tablas y figuras.

\section{RESULTADOS}

De los 345 sujetos analizados un $64,64 \%$ pertenece al tercer ciclo de primaria y un $35,36 \%$ pertenece al segundo ciclo de la E.S.O. Un 56,81\% de los encuestados son chicas, mientras que un $43,19 \%$ son chicos.

De los sujetos analizados el $97,5 \%$ desayuna mientras que el $2,5 \%$ no desayuna. Dentro de los que desayunan un $90,31 \%$ desayuna nada más levantarse y el 7,19\% desayuna a media mañana.

Un 79,17\% de los encuestados desayunan los 7 días de la semana, un 6,73\% desayuna 6 días, un 9,62\% desayuna 5 días y un $4,48 \%$ el resto de días.

En primaria un $37,22 \%$ de los sujetos analizados realizan un desayuno completo, es decir, ingieren lácteos, cereales y fruta o zumo mientras que un $62,78 \%$ no realiza un desayuno completo. En secundaria estos datos se agravan aún más, siendo un $23,77 \%$ de encuestados lo que realizan un desayuno adecuado en contra posición al 76,23\% que no realiza un buen desayuno.

$\mathrm{Al}$ diferenciar entre géneros se puede ver que hay un porcentaje mayor de chicos que de chicas que realizan un desayuno completo. Los chicos son un 35,57\% los que realizan un buen desayuno mientras que las chicas son un $30,1 \%$ las que realizan un buen desayuno. Las 
causas más comunes por las que los jóvenes no desayunan son la falta de tiempo, no se levantan con apetito y por falta de tiempo al levantarse con la hora justa por las mañanas.

En cuanto al lugar donde realizan el desayuno el $98,4 \%$ de los alumnos desayuna en casa, y dentro de esta el sitio donde suelen desayunar la mayoría de ellos es en la cocina. Sin embargo hay un 1,6\% que desayuna fuera de casa, más concretamente en el coche de camino a clase.
De todos los alumnos que rellenaron el cuestionario hay un $43,27 \%$ que realiza el desayuno solo, mientras que el 53,73\% restante realiza el desayuno acompañado en su mayoría por padres y hermanos.

En el gráfico 1 se puede observar aquellos grupos de alimentos que consumen los alumnos en función a las diferentes etapas y poder compararlos respecto al total de los alumnos.

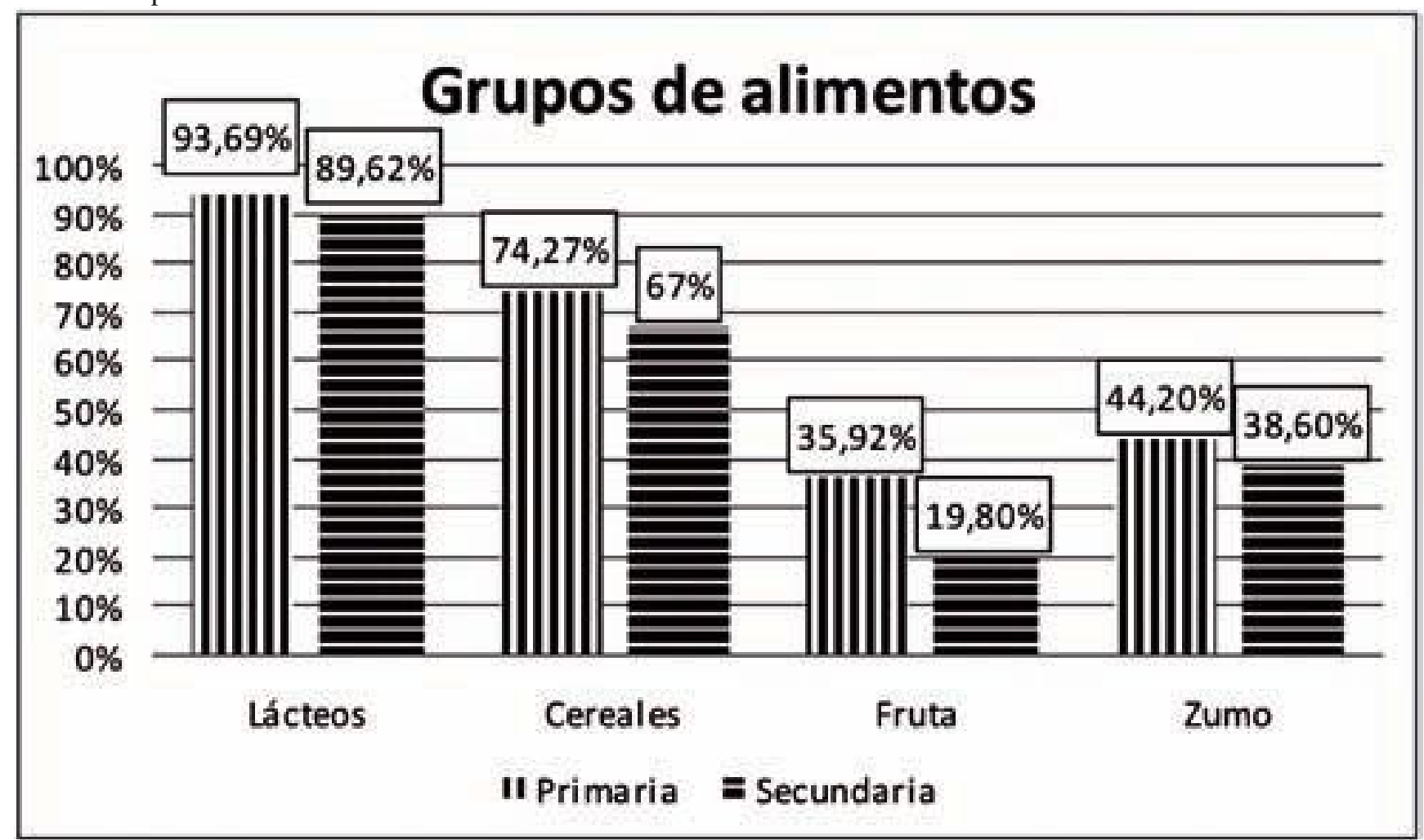

En el gráfico 1 se aprecia como el porcentaje de alumnos de primaria que ingieren lácteos es más elevado que en secundaria, aunque la diferencia es mínima. En cuanto a cereales seguimos observando que es superior esta ingesta en primaria que en secundaria con una diferencia mayor. En la fruta vemos como estos porcentajes bajan pero aun así es más consumida en primaria que en secundaria sin embargo la diferencia es considerable. Al igual que pasa con el zumo en primaria, etapa en la que se consume más que en secundaria. Por lo que se puede ver que se desayuna mejor en primaria que en secundaria.

En la tabla 1 se refleja las diferentes variables cuantitativas en relación al tiempo empleado para la ingesta del desayuno.

Tabla 1. Variable Tiempo

\begin{tabular}{cccc} 
& Primaria & Secundaria & Total \\
\hline Media & 9,97 & 8,52 & 9,31 \\
Desviación Típica & 5,06 & 4,75 & 5,03 \\
Moda & 10,00 & 5,00 & 5,00 \\
Máximo & 21,00 & 20,00 & 21,00 \\
Mínimo & 1,00 & 1,00 & 1,00 \\
\hline
\end{tabular}


En la tabla 1 se puede observar como el tiempo medio dedicado al desayuno es de 9 minutos y 18 segundos. En primaria se le dedica más tiempo al desayuno algo lógico si tenemos en cuenta que en esta etapa hemos visto que desayunan mejor los jóvenes. Otro dato llamativo es que el menor tiempo dedicado al desayuno es de un minuto, casi siempre asociado a la ingesta de leche mientras que el mayor tiempo dedicado fue de 21 minutos asociado a un completo desayuno.

Se ha visto que la importancia de conocer las características del desayuno son mayores en el género femenino (con una media de 7,97 sobre 10 en una escala numérica) que en el masculino (con una media de 7,62 sobre 10 en una escala).

Por otro lado en primaria esta inquietud es mayor $(8,45$ sobre 10$)$ que en secundaria $(6,71$ sobre 10$)$.

\section{DISCUSIÓN}

El objetivo de este estudio fue conocer las características del desayuno en alumnos de Educación obligatoria, teniendo en cuenta, el tiempo que le dedican, la compañía con la que lo realizan o los alimentos que incluyen en su desayuno.

El primer dato que debemos de tener en cuenta es el de jóvenes que desayunan, 90,31\% que puede parecer positivo y que además coincide con los datos obtenidos por Dura (2002), en el que el 93,2\% de los encuestados desayunaban nada más levantarse. En cuanto al porcentaje de alumnos que no desayunan también son similares con un 2,5\% obtenido en este estudio y un $3 \%$ obtenido en el de Durá (2002).

Sin embargo estos datos no son un buen indicador para considerar quienes realizan un buen desayuno debido a que el $90 \%$ o más de los sujetos consumen solo lácteos o lácteos y otros alimentos. Pero en un estudio realizado por Diez-Gañan, Galán, León y Zorrilla (2008) se observó que el 96,4\% de los sujetos consumían lácteos solos o acompañados de galletas, cereales o bollería por lo que se puede ver que ha habido un pequeño descenso en el consumo de lácteos.

Se debe de considerar como aspecto clave el lugar donde se realiza el desayuno siendo un $98,4 \%$ los alumnos que desayunan en casa y concretando más en el lugar en la cocina. Datos que se aproximan a los obtenidos por Diez-Gañan, Galán, León y Zorrilla (2008), los cuáles muestran que el 96\% desayunaba en casa.

Se debe tener en cuenta que $79,17 \%$ de los encuestados desayunan los 7 días de la semana, mientras que un $9,62 \%$ desayuna 5 días o menos debido a que los fines de semana son los días que menos desayuna los jóvenes. Entre las justificaciones más comunes encontramos la falta de hambre o la falta de tiempo, al levantarse tarde y juntarse con la hora de comer.

Según el estudio realizado por Amat et al (2006) en el que se realizó un estudio descriptivo sobre los hábitos alimentarios de los preadolescentes de Viladecans, se vio que las niñas son el grupo que menos desayuna cada día y por lo tanto que peor desayuna. Coincidiendo con los datos obtenidos en los que se puede ver que el $30,10 \%$ realizan un correcto desayuno, frente al $35,57 \%$ de nińos que desayunan bien, datos que tienden a emporar con el paso de primaria a secundaria.

\section{CONCLUSIONES}

Los alumnos de primaria realizan mejor el desayuno que los de secundaria ya el 37,22\% ingieren lácteos, cereales y fruta o zumo, mientras que en secundaria este porcentaje se reduce hasta el 23,77\%. Los chicos desayunan mejor que las chicas aunque esta diferencia no es demasiado amplia.

Para considerar que es un buen desayuno hay que tener en cuenta que desayunen en casa, requisito que se cumple, el $98,4 \%$ de los alumnos desayuna en casa.

En cuanto al tiempo dedicado 9 minutos y 18 segundos, es poco tiempo ya el tiempo recomendado para el desayuno es entre 15 y 20 minutos, por lo tanto es un aspecto sobre el que se debe de incidir más. Aunque las causas más utilizas para justificar este tiempo es debido a que no se levanta con el suficiente tiempo, o no se levanta con apetito.

El 53,73 \% desayuna acompañado por sus padres y hermanos, mientras que el $43,27 \%$ desayuna solo aspecto negativo para que el desayuno sea considerado "ideal". El desayuno tiene que realizarse acompañado por la familia para que el ambiente sea más adecuado.

Es llamativo que las chicas le dan más importancia al desayuno, con una media de 7,97 sobre 10 ya que desayunan peor que los chicos. Estos le dan menos importancia al desayuno con un 7,62 de media sobre 10 , sin embargo son los que mejor desayuno realizan.

Otro dato preocupante es el que se puede apreciar en cuanto a la importancia que le dan a estos contenidos, debido a que en primaria le dan una media de 8,45 sobre 10 , mientras que en secundaria desciende drásticamente hasta 6,7 de media sobre 10. Estos datos concuerdan con los referidos a la mejor ingesta de alimentos según la etapa de educación obligatoria obtenidos a lo largo de este estudio.

Para líneas de investigación posteriores se recomienda encuestar a los padres, para conocer su opinión respecto al desayuno, si creen que sus hijos desayunan correctamente o si se involucran tanto en la realización como en la ingesta del desayuno.

\section{APLICACIONES PRÁCTICAS}

El centro de actuación debería de ser en los centros escolares, tanto de primaria como de secundaria. En los centros escolares que dispongan de comedor escolar o cantina no deberían de limitarse a ofertar una comida al mediodía, sino que también ofrezcan y recomiendan 
la posibilidad de que los alumnos del centro realicen la primera comida del día en el comedor escolar. De esta manera se asegura que ingieran un desayuno ideal o equilibrado, realizado siempre por el especialista del centro.

En aquellos centros donde no dispongan de comedor, se podrían trabajar los contenidos relacionados con la nutrición y el desayuno en las clases de Educación física o en tutoría. También sería importante, por parte de los centros, organizar charlas informativas impartidas

\section{REFERENCIAS}

Amat, M.A., Anunciaby, V., Soto, J., Alonso, N., Villalmanzo, A. y Lopera,S.(2006). Estudio descriptivo sobre hábitos alimentarios en el desayuno y almuerzo de los preadolescentes de Viladecans (Barcelona). Nure Investigación, 23.

Derufino, P., Redondo, C., Amigo, T., GonzálezLamuño, D., García, M. y Grupo AVENA (2005). Desayuno y almuerzo de los adolescentes de los escolarizados de Santander. Nutrición hospitalaria, 20 (3), 217-222.

Diez-Gañan, L., Galán, I., León, C., y Zorrilla. (2008). Encuesta de Nutrición infantil de la comunidad de Madrid. Madrid: Consejería de sanidad de la comunidad de Madrid.

Durá, T. (2002). El desayuno de los alumnos de por nutricionistas, haciéndoles ver la importancia del desayuno y los beneficios tanto a nivel físico como intelectual. Hacerles ver que no solo es importante lo que desayunan, sino con quien desayunan, dónde y cuánto tiempo le dedican.

Colocar carteles por los centros con las imágenes de deportistas o personajes públicos a los que admiran haciéndoles ver, que ellos realizan un buen desayuno para poder afrontar el día llenos de energía.

Educación Secundaria Obligatoria (ESO). Nutrición Hospitalaria, 17 (4), 189-196.

Galiano, M.J. y Moreno, J.M. (2010). El desayuno en la infancia: más que una buena costumbre. Acta pediatría Española, 68 (8), 403-408.

González, M.P., y De la Rosa, V. (2007). Los adolescentes que consumen comida rápida y no desayunan tienen más riesgo de ser obesos en la vida adulta. Evidencias en Pediatría, 3 (46).

Herrero, R., y Fillat, J.C. (2006).Estudio sobre el desayuno y el rendimiento escolar en un grupo de adolescentes. Nutrición Hospitalaria, 21 (3), 346-352.

Núñez, G., Mazzitelli, C., y Vázquez, S. (2007). ¿Qué saben nuestros alumnos sobre alimentación y nutrición?. Revista Iberoamericana de Educación, 43 (5), 1-8. 